\title{
Bit-rate-transparent optical RZ-to-NRZ format conversion based on linear spectral phase filtering
}

Maram, Reza; Da Ros, Francesco; Guan, Pengyu; Røge, Kasper Meldgaard; Galili, Michael; Oxenløwe, Leif Katsuo; Azana, Jose

Published in:

Optical Fiber Communication Conference 2017

Link to article, DOI:

10.1364/OFC.2017.Th4l.1

Publication date:

2017

Document Version

Peer reviewed version

Link back to DTU Orbit

Citation (APA):

Maram, R., Da Ros, F., Guan, P., Røge, K. M., Galili, M., Oxenløwe, L. K., \& Azana, J. (2017). Bit-ratetransparent optical RZ-to-NRZ format conversion based on linear spectral phase filtering. In Optical Fiber Communication Conference 2017 [Th4l.1] Optical Society of America (OSA).

https://doi.org/10.1364/OFC.2017.Th4l.1

\section{General rights}

Copyright and moral rights for the publications made accessible in the public portal are retained by the authors and/or other copyright owners and it is a condition of accessing publications that users recognise and abide by the legal requirements associated with these rights.

- Users may download and print one copy of any publication from the public portal for the purpose of private study or research.

- You may not further distribute the material or use it for any profit-making activity or commercial gain

- You may freely distribute the URL identifying the publication in the public portal 


\title{
Bit-rate-transparent optical RZ-to-NRZ format conversion based on linear spectral phase filtering
}

\author{
Reza Maram, ${ }^{1}$ Francesco Da Ros, ${ }^{2}$ Pengyu Guan, ${ }^{2}$ Kasper Meldgaard Røge, ${ }^{2}$ Michael Galili, ${ }^{2}$ Leif Katsuo \\ Oxenløwe, ${ }^{2}$ and José Azaña ${ }^{1}$ \\ ${ }^{1}$ Institut National de la Recherche Scientifique-Énergie, Matériaux et Télécom., Montréal, QC H5A 1K6, Canada \\ ${ }^{2}$ DTU Fotonik, Department of Photonics Engineering, Technical University of Denmark, 2800 Kgs. Lyngby, Denmark \\ *Corresponding author: r.maram.q@emt.inrs.ca
}

\begin{abstract}
We propose a novel and strikingly simple design for all-optical bit-rate-transparent RZto-NRZ conversion based on optical phase filtering. The proposed concept is experimentally validated through format conversion of a $640 \mathrm{Gbit} / \mathrm{s}$ coherent RZ signal to NRZ signal.

OCIS codes: (060.2330) Fiber optics communications; (070.2615) Frequency filtering; (200.4560) Optical data processing
\end{abstract}

\section{Introduction}

Optical format conversion is an important functionality in nodes interfacing optical communication networks operating with different modulation formats [1,2]. Format conversion between return-to-zero (RZ) and non-returnto-zero (NRZ) on-off keying (OOK) formats is one essential conversion, since both formats are mature and widely used in different parts of the networks, e.g., the RZ format is typically adopted in time division-multiplexing (OTDM) systems to be able to perform pulse multiplexing, while the NRZ format is preferred in wavelength-divisionmultiplexing (WDM) systems due to its higher spectral efficiency [1,2]. Schemes based on linear optical signal processing for RZ-to-NRZ format conversion have attracted great interest due to their intrinsic simplicity, suitability for high speed operation and stable performance. Present linear RZ-to-NRZ format converters rely on the use of a periodic amplitude or phase filter with a free spectral range (FSR) precisely fixed to twice the bit rate of the incoming RZ data signal [1-3]. However, this method requires accurate adjustment of the filter FSR for different bit rates and therefore is bit-rate dependent. In addition, fabrication of such high-precision periodic filters is challenging due to practical fabrication errors [4].

In this paper, we propose and theoretically analyze the performance of a new, strikingly simple concept to perform RZ-to-NRZ conversion based on spectral phase filtering of the carrier frequency component of the incoming RZ data signals. A key advantage of the proposed format converter is that it removes the bit-rate dependency and realizes highQ-factor format conversion over a wide bit-rate range, without requiring any additional tuning or adjustments, thus significantly simplifying the filter design and implementation. Further, we experimentally demonstrate the desired format conversion at $640 \mathrm{Gbit} / \mathrm{s}$ by manipulating the spectral phase of a coherent RZ signal using a commercial linear optical waveshaper.

\section{Operation Principle and Simulation Results}

The operation principle of the proposed approach is illustrated through the results in Figs. 1(a)-(f), which show numerical simulations of the evolution of the input RZ signal through the conversion system both in the frequency domain (Figs. 1(a)-(c)) and in the time domain (Figs. 1(d)-(f)). Figs 1(a) and (d) show the spectrum and eye diagram of an input RZ data signal with a pseudorandom bit sequence (PRBS) of $2^{7}-1$, FWHM of $720 \mathrm{fs}$ and bit rate of 640 Gbits. In Fig. 1(d), the red curve shows the spectral phase of the used phase filter with a spectral line-width of $F_{b}=9$ GHz. This phase filter $\pi$-phase-shifts the carrier frequency component of the RZ signal with respect to the rest of the signal spectrum. In Fig. 1(e), the solid blue curve shows the spectrum of the RZ signal after phase filtering: the filtered energy spectrum remains unchanged with respect to that of the input signal. However, the time-domain eye diagram (shown in Fig. 1(b)) reveals the successful conversion from RZ to NRZ. It is worth mentioning that the amplitude ripples of the converted NRZ signal after the phase filter are significantly lower than those typically obtained using present amplitude/phase filtering approaches [1-3]. The quality of the converted NRZ signal can be further enhanced by an additional optical band-pass filter (OBPF), to remove spectral components associated to the fast temporal features that can be observed in the base-line of the eye diagram shown in Fig. 1(b). The 3-dB bandwidth of the Gaussian-shape OBPF used in this example is $820 \mathrm{GHz}$, as depicted in Fig 1(e). Figs. 1(c) and (f) show the eye diagram 
and spectrum of the final converted NRZ signal after filtering by the OBPF.
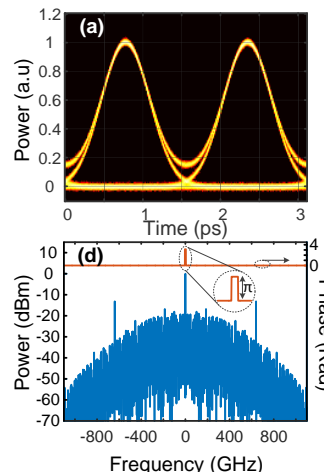

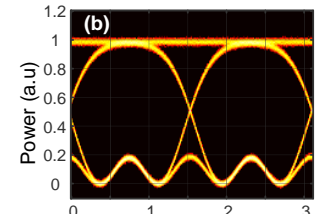

Time (ps)

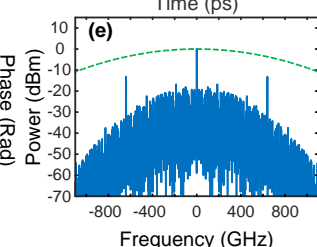

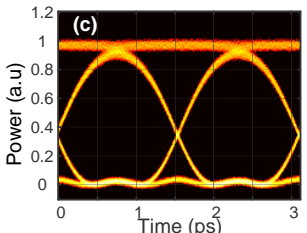

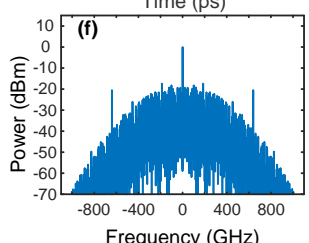

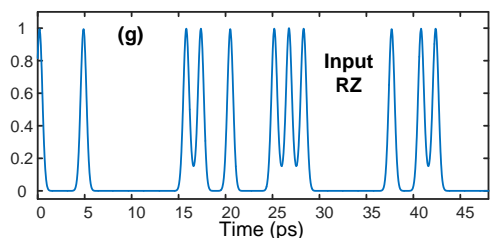

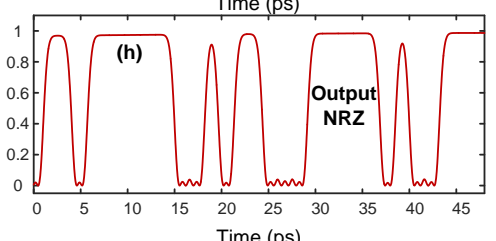

Fig. 1. Numerical simulation results. (a)-(f): Spectra and eye diagrams evolution of an input RZ signal through a format converter from RZ to NRZ at $640 \mathrm{Gbit} / \mathrm{s}$. (a) and (d) are the spectrum and eye diagram of the input RZ signal. (b) and (e) are the spectrum and eye diagram of the RZ signal filtered by the phase filter (PF) shown in (d) (red curve). (c) and (f) are the spectrum and eye diagram of the converted NRZ signal,

following OBPF with the filter shown in (e) (green curve). (g) and (h) show the temporal traces of the input and output data signals.

Notice that in this scheme, the bit pattern of the input data signal is also inverted at the output data signal, performing a logic NOT gate operation, as shown in Figs. 1(g) and (h). The defined phase filter can be practically implemented using a variety of technologies, such as a line-by-line optical pulse shapers (used in the experiments reported here), fiber Bragg gratings (FBGs), thin film filters, or photonic ring resonators, among others [3].
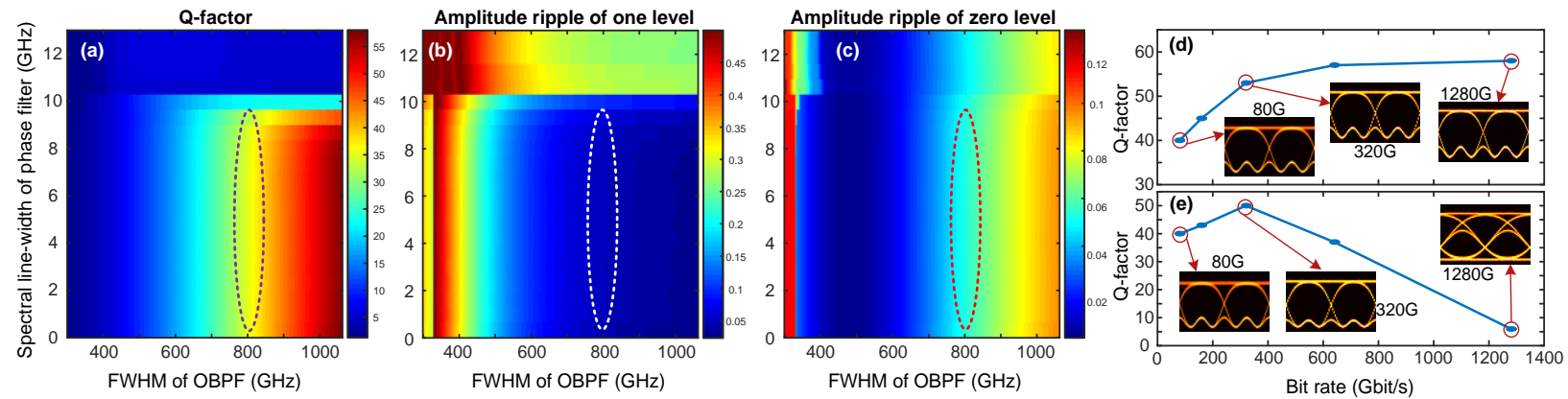

Fig. 2. (a) Q-factor, and (b) and (c) amplitude ripple of one and zero levels of the converted NRZ versus spectral line-width of the PF and bandwidth of the OBPF for $640 \mathrm{Gbit} / \mathrm{s} \mathrm{RZ-to-NRZ} \mathrm{format} \mathrm{conversion.} \mathrm{(d)-(c)} \mathrm{Q-factor} \mathrm{versus} \mathrm{operating} \mathrm{bit-rate} \mathrm{for} \mathrm{fixed} \mathrm{filtering} \mathrm{characteristics.}$

For a good format conversion, both a high Q-factor (defined as $\mathrm{Q}=\left(\mathrm{P}_{1}-\mathrm{P}_{0}\right) /\left(\sigma_{1}+\sigma_{0}\right)$, where $\mathrm{P}_{1}$ and $\mathrm{P}_{0}$ are the means and $\sigma_{1}$ and $\sigma_{0}$ denote the standard deviations of the logic "one" and "zero" levels, respectively) and low amplitude ripples (defined as the peak-to-peak power deviation of the high and low levels for the logic "one" and "zero", respectively, in the converted NRZ normalized eye diagram) are required for the converted signal [2]. Figs 2(a)-(c) analyze the Q-factor and amplitude ripples of the converted NRZ signal as a function of spectral line-width of the phase filter and the OBPF 3-dB bandwidth at a bit-rate of $640 \mathrm{Gbit} / \mathrm{s}$. As can be inferred from Figs 2(a)-(c), the linewidth of the phase filter $\left(\mathrm{F}_{\mathrm{b}}\right)$ should be narrower than $\sim 10 \mathrm{GHz}$ for high-quality format conversion. One can also find that a higher Q-factor and lower one-level amplitude ripple can be achieved as the OBPF's bandwidth is increased (Figs.2 (a) and (b)). Indeed, the highest Q-factor ( 55) and the lowest one-level amplitude ripple are already obtained using only the phase filter. However, in order to reduce the amplitude ripple of the zero-level to its minimum value, an additional OBPF of $\sim 500 \mathrm{GHz}$ bandwidth is required. Therefore, there is a trade-off between Q-factor and zerolevel amplitude ripple. An OBPF with a bandwidth around $820 \mathrm{GHz}$ will result in an optimum RZ-to-NRZ format conversion performance, with a Q-factor of $\sim 36$, corresponding to the ellipses in Figs. 2(a)-(c).

Finally, it should be pointed out that since this technique relies only on spectral phase manipulation of the input carrier component, even if the operating bit-rate varies, the relative positions of the phase filter and carrier frequency of the RZ spectrum remains unchanged. Therefore, this technique is transparent to the input bit-rate. This key feature is illustrated in Figs. 2(d) and (e), where the designed phase filter for a $640 \mathrm{Gbit} / \mathrm{s} \mathrm{RZ}$-to-NRZ format conversion (for example, $F_{b}=1 \mathrm{GHz}$ and FWHM of OBPF $=820 \mathrm{GHz}$ ) is tested for different input bit rates. Fig. 2(d) shows the case 
when only the phase filter is employed for the format conversion. This results in Q-factors greater than 40 as well as wide open eye diagrams over a wide range of input bit rates (well over Tbit/s). Inserts are eye-diagrams of the converted NRZ data signals at 80,320 , and $1280 \mathrm{Gbit} / \mathrm{s}$, respectively. Fig. 2(e) shows the case when an OBPF of $820 \mathrm{GHz}$ bandwidth is employed after the phase filter. Indeed, from the simulation results, we estimate that the optimum OBPF bandwidth for a given bit rate is $\sim 1.3$ times the bit rate. Therefore, in this example, the Q-factor will drop significantly for the bit-rate of $1280 \mathrm{Gbit} / \mathrm{s}$.

\section{Experimental Results}

In this section, we report a proof-of-concept experiment to validate the proposed method by performing RZ-to-NRZ conversion at a bit rate of $640 \mathrm{Gbit} / \mathrm{s}$. Fig. 3(a) presents the experimental setup for the target format conversion. The transmitter block generates a coherent RZ data signal at $640 \mathrm{Gbit} / \mathrm{s}$ [3] and delivers the generated data signal to the format conversion sub-system, comprising the proposed phase filter and OBPF. A line-by-line pulse shaper (Finisar WaveShaper 4000S) has been programmed to produce the needed filtering specifications, i.e., a spectral phase filter with $F_{b}=13 \mathrm{GHz}$ and a Gaussian amplitude filter with 820GHz FWHM. The spectra of the $640 \mathrm{Gbit} / \mathrm{s}$ input RZ and output NRZ signals, together with the spectral phase profile of the phase filter, are depicted in Fig. 3(b). As mentioned above, the output signal spectrum is identical to that of the input signal, except for the fact that the output signal's spectrum is slightly narrower owing to the OPBF. Fig. 3(c) and (d) illustrate the temporal intensity traces of (c) the $640 \mathrm{Gbit} / \mathrm{s}$ input RZ data signal and (d) the converted NRZ signal at the output, measured by a 500-GHz bandwidth optical sampling scope; the insets are measured in averaging mode. As can be clearly seen, the proposed concept has implemented the target RZ-to-NRZ format conversion. It should be noted that the spectral line-width of the phase filter, which is determined by the minimum bandwidth of the employed waveshaper, is relatively far from the predicted optimal regions shown in Figs 2(a)-(c), therefore the output NRZ exhibits an amplitude modulation. Finally, BER measurements are carried out to analyze the quality of the time-demultiplexed data of the input and output signals. Fig. 3(e) shows receiver sensitivities at a BER of $10^{-7}$ for all 64 channels of the input (blue circle) and output (red square) signals. In average, the output signal has the same receiver sensitivity as the input signal.
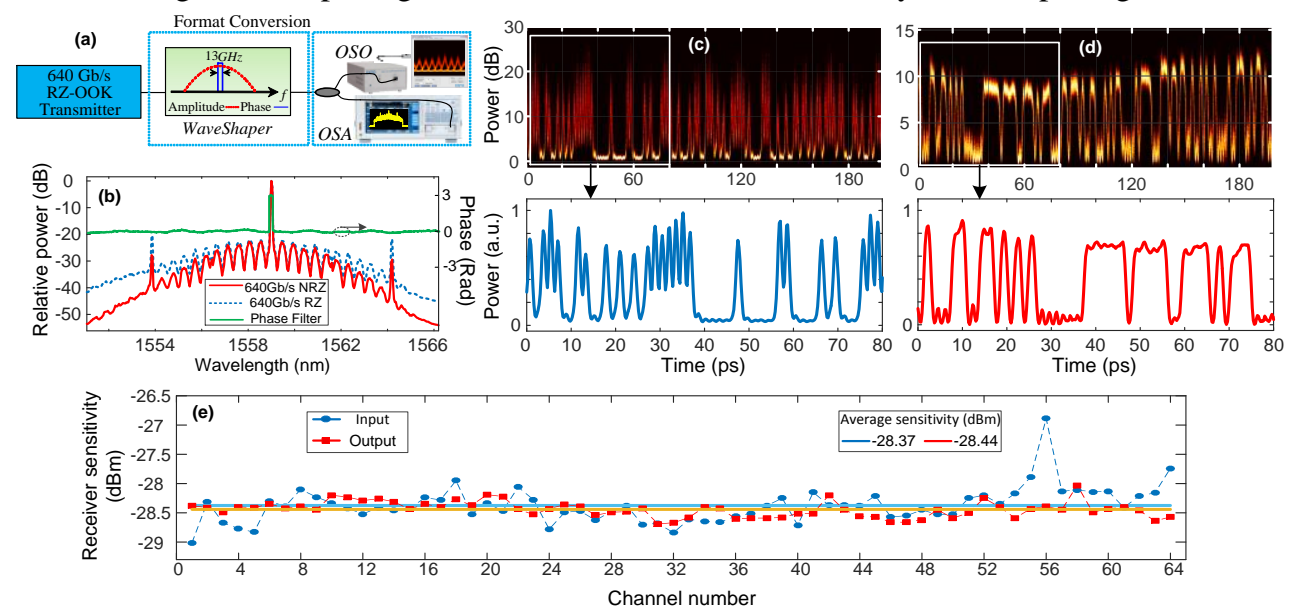

Fig. 3. (a) Experimental setup; (b) Spectra of the input (blue) and output (red) signals as well as spectral phase profile of the phase filter (green); (c) and (d) Measured temporal waveforms of the input RZ and output NRZ signals, respectively. (c) Receiver sensitivities of all channels.

\section{Acknowledgment}

Authors acknowledge the Natural Sciences and Engineering Research Council of Canada (NSERC), Danish Research Council FTP project TOR (ref. no. 12-127224), the DFF Sap. Aude Adv. Grant NANO-SPECs (DFF-4005-00558B) and the DNRF Research Centre, SPOC, (ref. DNRF123).

\section{References}

[1] M. Xiong et al, "Simultaneous RZ-OOK to NRZ-OOK and RZ-DPSK to NRZ-DPSK format conversion in a silicon microring resonator," Opt. Express 20, 27263-27272 (2012).

[2] Y. Ding et al, “Generation of a $640 \mathrm{Gbit} / \mathrm{s}$ NRZ OTDM signal using a silicon microring resonator,” Opt. Express 19, 6471-6477 (2011).

[3] R. Maram et al, "640 Gbit/s return-to-zero to non-return-to-zero format conversion based on optical linear spectral phase filtering," Opt. Lett. 41, 64-67 (2016).

[4]. L. Chrostowski et al, "Impact of fabrication non-uniformity on chip-scale silicon photonic integrated circuits," Optical Fiber Communication (OFC) Conference, San Francisco, US, (2014). 